\title{
Sociedad del conocimiento y las TEPs
}

\section{Knowledge society and TEPs}

Econ. Fernando José Zambrano Farías, MEF

Universidad Internacional del Ecuador, Ecuador

Ing. Com. Karen Andrea Balladares Ponguillo, MAE

Universidad de Guayaquil, Ecuador

Autor para correspondencia: fezambranofa@uide.edu.ec, karen.balladaresp@ug.edu.ec Fecha de recepción: 02 de Agosto de 2017 - Fecha de aceptación: 30 de Septiembre de 2017

\section{Resumen}

Las Tecnologías del Empoderamiento y la Participación, desde ahora TEP, es una nueva terminología que se le asigna a las tecnologías que se utilizan como sustento para la cohesión social de un grupo determinado de personas. Hablamos de una red social en la cual se fomenta la participación activa de los usuarios en temas relacionados con política, sociedad y/o educación Gracias a las nuevas tecnologías se puede organizar, registrar, almacenar y compartir en tiempo real todos los cambios que se están viviendo y participar de una manera activa en ellos sin necesidad de pertenecer a un grupo organizado o estar cursando algún programa académico específico.

Palabras Claves: sociedad del conocimiento; TIC; TAC; TEP

\begin{abstract}
The Technologies of Empowerment and Participation, now TEP, is a new terminology that is assigned to the technologies that are used as sustenance for the social cohesion of a certain group of people. We speak of a social network in which the active participation of users in topics related to politics, society and / or education is encouraged Thanks to new technologies you can organize, record, store and share in real time all the changes that are being made. Living and participating in an active way in them without needing to belong to an organized group or to be studying a specific academic program.
\end{abstract}

Key words: knowledge society; ICT; TAC; TEP 


\section{Introducción}

Para los antiguos el tiempo estaba representado por los eventos cíclicos de la naturaleza, los seres humanos no se preocupaban por medirlo, la gente no interesaba por llevar la cuenta de su edad, pero a finales de edad media empieza a construirse grandes maquinarias de relojería, los encuentros se podían hacer con el sonar de las campanas. En la revolución industrial el reloj tuvo una influencia mayor que la máquina a vapor, porque influyó en la organización de la vida de la sociedad occidental, la sociedad industrial incorporó nuevas formas de pensar los procesos económicos y sociales, las teorías económicas relacionaban las nociones de tierra, capital y trabajo. El capital era el recurso de producción crítico. Capital y trabajo eran conceptos totalmente separados y en oposición.

A mediados de los 70 la convergencia tecnológica da a lugar al progresivo desarrollo de las computadoras, cuando esas tecnologías se trasladan desde los sectores militares a los civiles el efecto en la sociedad se hace evidente, el futuro ya está aquí y es digital. Gran parte de los elementos de nuestra vida analógica son convertidos en 1 y 0 , nace el paradigma de la red. El intercambio de objetos, muta al intercambio de la información y la gestión de la información pasa a ser vital en los procesos económicos, a diferencia de la materia construida por átomos. Los bites no se pierden, circulan y se reutilizan constantemente.

A principios de los 90 Peter Braker introduce la noción de la sociedad del conocimiento, la información interpretada se transforma en conocimiento y ese conocimiento es recurso clave, es más importante que el trabajo, la tierra y capital. Aparecen nuevos mecanismos de integración de individuos, nuevas formas de construir identidad de personal a partir de una red. El conocimiento de los patrones genéticos opera sobre la salud, alimentación y el medio ambiente. La sociedad pos capitalista es una sociedad del conocimiento. Y quien tenga conocimiento de gestión sobre los procesos tiene la producción. El conocimiento puede relocalizar procesos y conseguir capitales en cualquier sitio del mundo interconectado. El conocimiento reside en la persona, no puede ser comprado con dinero ni creado por capitales de inversión. Se extiende a todas las áreas de la actividad humana. Estamos al inicio de una revolución de la información y no es tecnológica, cambiando la percepción del espacio y del tiempo, dimensiones fundamentales de la experiencia humana. No se trata de nuevas máquinas, software. Es una revolución de conceptos, donde tenemos que muy rápidamente conseguir y procesar información. La sociedad del conocimiento cambiará por completo las estructuras sociales, la forma de comportarnos y dirigirnos.

\section{Materiales}

La metodología de investigación aplicada es de tipo documental y descriptiva. Según Arias (1995) señala que la investigación documental, "es un proceso basado en la búsqueda, recuperación, análisis, critica e interpretación de datos secundarios, es decir, los obtenidos y registrados por otros investigadores en fuentes documentales: impresas, audiovisuales o electrónicas". (p. 25). Soportada bajo una revisión documental de trabajos, estudios de sociedad del conocimiento, tendencias de las tecnologías de empoderamiento y participación, con el apoyo de artículos de google académico, revistas indexadas, lo que permitió realizar el análisis del mismo. 
La técnica utilizada en el estudio fue la de fichaje, es una forma de recolectar y analizar información proveniente de la investigación documental y bibliográfica. El análisis secundario según Hernández consiste en "utilizar datos recolectados por otros investigadores... En este caso es necesario tener la certeza de que los datos son válidos y confiables, así como conocer la manera como fueron codificados." (1997). Los núcleos teóricos que se van a investigar serían la sociedad del conocimiento y las tecnologías de empoderamiento y participación. Los pasos para la investigación son: primero hacer una revisión de toda la información secundaria; el segundo paso, la selección del material bajo estudio; el tercer paso, analizar los documentos y resumir lo observado; y por último redactar el informe de investigación.

\section{Resultados}

La noción de "sociedad del conocimiento" (knowledge society) emergió hacia finales de los años 90; es empleada particularmente en medios académicos, como alternativa que ciertos prefieren a "sociedad de la información".

La UNESCO, en particular, ha adoptado el término "sociedad del conocimiento", o su variante, "sociedades del saber", dentro de sus políticas institucionales. Ha desarrollado una reflexión en torno al tema, que busca incorporar una concepción más integral, no ligado solamente a la dimensión económica. Por ejemplo, Abdul Waheed Khan (subdirector general de la UNESCO para la Comunicación y la Información), escribe (2003): "Information society is the building block for knowledge societies. Whereas I see the concept of 'information society' as linked to the idea of 'technological innovation', the concept of 'knowledge societies' includes a dimension of social, cultural, economic, political and institutional transformation, and a more pluralistic and developmental perspective. In my view, the concept of 'knowledge societies' is preferable to that of the 'information society' because it better captures the complexity and dynamism of the changes taking place. (...) the knowledge in question is important not only for economic growth but also for empowering and developing all sectors of society".

Un matiz en este debate, que solo concierne a los idiomas latinos, es la distinción entre "conocimiento" o "saber" (ambos en inglés se traducen como "knowledge"). La noción de "saberes" implica certitudes más precisas o prácticas, mientras que conocimiento [1] abarca una comprensión más global o analítica. Por lo general, en este contexto se los utiliza indistintamente, si bien en español, al menos, conocimiento parece ser más usual.

Se trata en definitiva de conocer y de explorar los posibles usos didácticos que las TIC tienen para el aprendizaje y la docencia. Es decir, las TAC van más allá de aprender meramente a usar las TIC y apuestan por explorar estas herramientas tecnológicas al servicio del aprendizaje y de la adquisición de conocimiento. Podríamos definir a las TEP como aquellas Tecnologías que son aplicadas para fomentar la participación de los "ciudadanos" en temas de índole político o social generando de esta forma una especie de Empoderamientoy concientización de su posición en la sociedad que se traduce en expresiones de protesta y/o acción pública.

Tünnermann, C., (2000) orienta mejor en este nuevo escenario de la Educación 


\section{Continua:}

Es eso y mucho más. Es la integración de todos los recursos docentes de que dispone la sociedad para la formación plena del hombre durante toda su vida. Siendo así, la educación permanente tiene una raíz ontológica y es, como se ha dicho, "una respuesta a la condición humana y a eso que llamamos los signos de los tiempos. (p. 144).

La UNESCO (2008) afirmó: Hoy en día, los docentes en ejercicio necesitan estar preparados para ofrecer a sus estudiantes oportunidades de aprendizaje apoyadas en las TIC; para utilizarlas y para saber cómo éstas pueden contribuir al aprendizaje de los estudiantes. Capacidades estas que actualmente forman parte integral del catálogo de competencias profesionales básicas de un docente. (p.2).

De acuerdo al vídeo conferencia realizada por la profesora española, psicóloga social experta en cibercultura Dolors Reig: Denominada Sociedad Aumentada y Aprendizaje para la Organización de Estados Iberoamericanos (OEI) y el Instituto Iberoamericano de TIC y Educación (IBERTIC) donde aborda que el uso de las Tic (Tecnología de información y de la comunicación) han generado un cambio de nuestra sociedad, desde lo micro a lo más macro.

\section{TIC-TAC-TEP}

E igualmente afirma que existe un consenso para la mayoría de los educadores, que las tecnologías sociales fueron las herramientas para lograr una recuperación de la sociabilidad del ser humano, así mismo aumentó la interacción con la información y se fortalecieron las sociedades de participación. Reig afirma que hemos entrado en el momento de conceptualizar y trabajar como ciudadanos o educadores en las Tecnologías participativas.

Estas tecnologías participativas dan nacimiento a una nueva definición: Las TEP denominadas (Tecnologías de Empoderamiento y participación). El termino Empoderar, viene del calco del inglés to empower, que se emplea en textos de sociología política con el sentido de "conceder poder [a un colectivo desfavorecido] para que, mediante su autogestión, mejore sus condiciones de vida".

A partir de ello, para el caso de las TEP, concede el poder de la información desde diversas ópticas del conocimiento. Las TEP dan nacimiento a un nuevo universo, que apuesta a la participación y empoderamiento de los receptores, creando un puente entre el conocimiento individual y el colectivo, porque permite un aprendizaje pro-activo que potencia las habilidades individuales, en un contexto participativo. Las Tep además fomentan explotar las posibilidades de la inteligencia y las creatividades colectivas

La imagen resume el gran universo de individuos que conocen la respuesta y quieren tener una participación activa, para brindar su conocimiento, sin la necesidad de estar en el mismo sitio, espacio lugar o tiempo. Los avances de la tecnología permiten que los usuarios puedan participar y organizarse para crear ese conocimiento colectivo. Pero Reig nos lleva mucho más allá de saber usar las tecnologías participativas, (WEB 2.0, Facebook; Twitter, 
YouTube, etc.) nos lleva a la reflexión de ¿cuál sería el modelo ideal de educadores para aplicarla?

Nos Dice Que Las Tep, Están Implicando Una Ruptura, De Los Paradigmas De La Educación, Porque Se Rompen Los Formatos, Los Sistemas Educativos Diseñados Y Estructurados Para La Época De La Cultura Intelectual De La Ilustración. Las Tep Rompen Los Paradigmas De Conformar Las Clases Por Grupos De Edades O De Niveles De Aprendizajes.

Reig, propone dos roles de profesores:

$\checkmark$ El organizador de la inteligencia colectiva, quien orienta la obtención de productos de habilidades para organizarse.

$\checkmark$ Y un maestro experto en Participación, quien será el encargado de las habilidades de inteligencias, empoderamiento individual, creatividad colectiva.

El libro titulado Tecnologías de Información, Educación y Pobreza en América Latina: Escrito por Bertha Salinas Amescua, Laura M. Porras, Antonio Santos, José Ramos, plantea que los procesos que permiten la función pedagógica de empoderamiento de los individuos son los siguientes:

Autonomía: Fomentar las estrategias de empoderamiento INDIVIDUAL, a través de las TICs que desarrollen un sentido de independencia y confianza y autoeficacia que asume la responsabilidad de su propio aprendizaje y cuestiona libremente lo que aprende. (Knowes 1998).

Herramienta Cognitiva: Propiciar que el uso de las Tic, trascienda la finalidad instrumental y se convierta en una experiencia de enseñanza-aprendizaje, para desarrollar habilidades cognitivas, con solución de problemas.

Construcción Social Del Conocimiento: Diseñar ambientes de integración grupal, que permitan las libres negociaciones de significados y racionamientos que construyan el conocimiento, elaborado socialmente.

\section{Discusión}

Mucho se ha hablado en los últimos años de las TIC. Las Tecnologías de la Información y la Comunicación suponen la utilización en el colegio de herramientas digitales y la informatización de la gestión. Pero como suele ocurrir con los cambios, la puesta en marcha de las TIC en las aulas no avanza a la misma velocidad en todos los centros. No solamente por las dificultades presupuestarias, que las hay, sino también, en ocasiones, por las reticencias de algunos frente al entusiasmo de otros. Hemos pasado, pues, de estudiar las nuevas tecnologías a enseñar y estudiar con ellas. Y ese concepto de nuevas tecnologías de la información y la comunicación se convierte en Tecnologías para el Aprendizaje y el Conocimiento, de ahí las siglas TAC.

La idea de las TAC ya no se limita a enseñar a manejar el ordenador, la tableta o la pantalla digital, sino al concepto de gestionar el conocimiento y esas máquinas son meros 
instrumentos para transmitirlo y su manejo se da por conocido. Las TIC transmiten la información y las TAC la hacen accesible para los alumnos. Una vez más el papel del docente se vuelve imprescindible. Ya no sólo consiste en explicar una materia, como comentábamos en una entrada anterior, sino que el buen profesor debe de ilusionar al alumno, activar su curiosidad e inspirar el amor por el aprendizaje.

Y ese aprendizaje ya no solamente se realiza con libros o cuadernos, sino que también se hace a través de nuevas tecnologías cuyos contenidos y metodología debe de conocer el docente. Para ello debemos de facilitar al alumno la posibilidad de que localice por sí mismo la información que necesita, filtrando en esa inmensa red que es el ciberespacio, los datos relevantes que necesita.

La función del profesor cambia y del maestro con sus lecciones magistrales pasamos a un facilitador y supervisor del aprendizaje a través de canales que se mezclarán con los que propongan los propios alumnos para conseguir que los conocimientos, competencias y habilidades sean los adecuados a las necesidades de los alumnos, tanto a nivel individual como a nivel de clase. Pero, esta evolución no se detiene y ahora de nuevo, las corrientes más innovadores no se conforman con las TAC y creen que también deben de ser utilizadas las TIC como vínculo entre la escuela y las demandas de la sociedad.

Es el modelo que Dolors Reig ha llamado TEP (Tecnologías del Empoderamiento y la Participación). Esta experta considera a las TEP como un paso natural en la evolución del universo digital, basado en dos fenómenos: la participación y la influencia, pasando de los expertos a la inteligencia colectiva, construida a base de aportaciones de miles de personas. Hay quien considera que la tecnología unida al empoderamiento y la participación es el futuro y requiere un sistema educativo centrado más en el aprendizaje autónomo y con el foco puesto en el estudiante.

Ante esta sopa de siglas y con tantas innovaciones en el sistema educativo, no conviene olvidar las palabras de la autora de "The Complete Speakers Sourcebook", Eleanor Doan: "Unas buenas herramientas no hacen un excelente profesor, pero un excelente profesor sí emplea bien las herramientas".

Los tiempos seguirán cambiando y nuevas ideas seguirán surgiendo, la tecnología seguirá aumentando con más herramientas digitales a nuestro alcance. Por lo tanto debemos enfocarnos más en la realidad de nuestros estudiantes que los contenidos curriculares, los cuales la mayoría de las veces carecen de significación para el estudiante. Los contenidos curriculares se enfocan más en satisfacer unas metas impuestas que no tienen nada que ver con la realidad del entorno del estudiante. Nosotros como docentes debemos aprender que la enseñanza no debe encajonarse en un aula, el aprendizaje debe ir más allá o sea fuera de ella. Debemos buscar un balance en el cual incluyamos todas estos instrumentos de apoyo como lo son TIC-TAC-TEP que se nos ofrecen a través de la Web, e irlas incorporando en nuestros planes a diario sé que de esta manera veremos un cambio en nuestros estudiantes , seamos facilitadores, dejemos que nuestros estudiantes descubran por ellos mismos, pienso que así desarrollamos en ellos destrezas de pensamiento, porque son ellos los que decidirán como lo aprenden y cómo lo usarán, que mejor que ellos para saber cuáles son sus 
necesidades .Seamos parte de este nuevo siglo de la era digital, sin temor a los cambios y a los nuevos retos de estos tiempos. En la actualidad resulta de suma importancia comprender que formamos parte de la llamada Sociedad del conocimiento, por lo que comprender a cabalidad de qué se trata exactamente, será la base para una adecuada adaptación, desarrollo y colaboración.

De acuerdo al sociólogo y profesor Yoneji Masuda, al hablar de "Sociedad del conocimiento" nos referimos a una sociedad que crece y se desarrolla alrededor de la información y que por ende aporta al florecimiento general de la creatividad intelectual humana. Siendo así, se hace referencia a la creciente capacidad tecnológica, que permite replantear la estructura de las relaciones sociales, económicas, culturales y políticas, de acuerdo a los desarrollos existentes en la infraestructura de telecomunicaciones con el fin de obtener o compartir cualquier información de una manera rápida desde cualquier lugar y de la forma que se prefiera.

El término como tal fue utilizado por primera vez por Peter Ferdinand Drucker (Considerado filófoco de la administración del siglo XX) y más tarde profundizado por autores como Robin Mandel o Nico Stehr. Finalmente, la UNESCO, considera que el término Sociedad del conocimiento, apunta a transformaciones sociales, culturales y económicas en apoyo al desarrollo sustentable, siendo los pilares de esta sociedad, el acceso a la información para todos, la libertad de expresión y la diversidad lingüística.

Las tendencias actuales, aún en el proceso de la adaptación a la ya descrita sociedad del conocimiento, son aún contradictorias y no muestran una vía única: Por una parte se habla de la necesidad de la superespecialización del trabajador (cualquiera que sea el nivel en el que se desempeñe), peor por otra parte, lo que explicaría el éxito que ya tienen las carreras cortas, se afirma que en un futuro no muy lejano, las personas cursarán a lo largo de su vida, un promedio de 7 carreras diferentes, pues requerirán desarrollar competencias complementarias de distinta índole. Lo que nos lleva ahora a preguntarnos ¿Cuál será el papel del educador en esta sociedad?, ¿Cuáles han de ser sus competencias profesionales?

Ciertamente, no se trata de que un educador ejerza siete carreras profesionales sino más bien lo que tendrá que hacer es manejar competencias relacionadas con varias de ellas, como por ejemplo la pedagogía, como base profesional, la didáctica y administración como disciplinas procedimentales, la psicología, sociología y antropología como áreas auxiliares inmediatas y la filosofía junto con las tres últimas que le concederán competencias epistemológicas requeridas en la práctica docente.

Vemos entonces que lo mencionado adquiere relevancia por el hecho del nuevo papel que adquiere el docente como ente formador de quienes en la actualidad se encuentran inmersos hasta por cuestión generacional, en la sociedad del conocimiento, los alumnos.

Siendo así, afirmamos entonces que la educación en la sociedad del conocimiento dejará de ser la transmisora de información, sino que, sin abandonar su función formativa, ha comenzado a ser la administradora del conocimiento que produce el ser humano, es decir adquiere el papel de "gestor del conocimiento", por lo tanto, el desarrollo de estas nuevas funciones exige de los docentes una filosofía, estructura y proceder centrado en la persona, es 
decir en el alumno, quien deberá desarrollar competencias mucho más que obtener un objetivo cualitativo específico. Esto significa abandonar las formas verticales de la estructura para hacerlas más horizontales y flexibles, pero sobre todo mucho más participativas y adaptables al cambio permanente que exige una sociedad basada en el dinámico conocimiento humano que trabaja de la mano con las nuevas tecnologías.

\section{Bibliografía}

Aguaded, J.I. Y Tirado, R. (2010). Ordenadores En Los Pupitres: Informática Y Telemática En El Proceso De Enseñanza---Aprendizaje En Los Centros Tic De Andalucía. Pixel---Bit. Revista De Medios Y Educación. 36; 5---28. Extraído El 25 De Junio De 2010 Desde Http://Www.Sav.Us.Es/Pixelbit/Actual/1.Pdf

Cabero, J., Llorente, M.C. Y Gisbert, M. (2007): El Papel Del Profesor Y El Alumno En Los Nuevos Entornos Tecnológicos De Formación. En J. Cabero, Nuevas Tecnologías Aplicadas A La Educación. (262---291). Madrid: Mcgraw Hill.

Cebrián, M.; Ruiz, J. Y Rodríguez, J. (2007). Estudio Del Impacto Del Proyecto Tic Desde La Opinión De Los Docentes Y Estudiantes En Los Primeros Años De Su Implantación En Los Centros Públicos De Andalucía. Málaga, Universidad De Málaga: Grupo De Investigación Nuevas Tecnologías Aplicadas A La Educación.

Cohen, L. \& Manion, L. (1990). Métodos De Investigación Educativa (Pp.271). Madrid: La Muralla.

Colás, M. P. Y Buendía, L. (1998). Investigación Educativa. 3ª Edición. Sevilla: Alfar. Departament D'educació.

Generalitat De Catalunya (2005). Pla Marc De Formación Permanente 2005---2010. Extraído El 25 $\begin{array}{llll}\text { De Junio } & \text { De } & \text { Desde }\end{array}$ Http://Www10.Gencat.Cat/Gencat/Binaris/Formacio_Permanent_Tcm33---21897.Pdf

Departament D’educació. Generalitat De Catalunya (2005). Pla Marc De Formació Permanent 2005--2010. Extraído El 25 De Junio De 2010 Desde Http://Www.Xtec.Cat/Formacio/Pla_Marc/Plamarc_Formacio.Pdf

Departament D'educació. Generalitat De Catalunya (2009). Organització Ifuncionament Dels Centres Educatius Públics D'educació Infantil I Primària I D’educació Especial. Curs 2009---2010. 43---47. Extraído El 25 De Junio De 2010 Http://Educacio.Gencat.Net/Portal/Pls/Portal/Ide_Utils_Pkg.Download_Fitxer?P_File=1041739 24435146677

Espuny, C. (2008). Disseny, Implementació I Avaluació Del Pla D’explotació De Recursos Tic Als Centres De Primària Del Baix Ebre. Tesis Doctoral. Tesis Doctorals En Xarxa. Extraído El 25 De Mayo De 2010 Desde Http://Www.Tesisenxarxa.Net/Tdx---0316109---123203

Espuny, C.; Gisbert, M. Y Coiduras, J. (2010). La Dinamización De Las Tic En Las Escuelas. Edutec: Revista Electrónica De Tecnología Educativa (32), 1---16. Extraído El 25 De Mayo De 2010 Desde

Http://Edutec.Rediris.Es/Revelec2/Revelec32/Dinamizacion_Tic_Escuelas.Html Issn: 1135--9250 
Garrido, M. C., Fernández, R., Y Sosa, J. M. (2008). Los Coordinadores Tic En Extremadura. Análisis Legislativo Y Valoración De Su Implantación En Los Centros Educativos De Primaria Y Secundaria De La Región. Quaderns Digitals. Eduteka. Extraído El 25 De Junio De 2010 Desde Http://Www.Eduteka.Org/Pdfdir/Descripcioncargocoodinador.Pdf

Fernández, S. Y Lázaro, M. N. (2008). Coordinador/A Tic. Pieza Clave Para La Integración De Las Nuevas Tecnologías En Las Aulas. Revista Latinoamericana De Tecnología Educativa, 7 (2), 177---187.

$$
\begin{array}{llllll}
\text { Extraído } & \text { El } & 2 & \text { De Julio De } 2010 \text { Desde }
\end{array}
$$

Http://Campusvirtual.Unex.Es/Cala/Editio/Index.Php?Journal=Relatec\&Page=Article\&Op=View \&Path [] =433\&Path [] =367 Ley Orgánica 2/2006, De 3 De Mayo, De Educación. Boe 106. Extraído El 12 De Junio De 2010 Desde

Http://Www.Educacion.Es/Dctm/Mepsyd/Horizontales/Prensa/Documentos/2008/Loe.Pdf?Do Cumentid=0901e $72 \mathrm{~b} 80027758$

Marquès, P. (2007). 5 Claves Para Una Buena Integración De Las Tic En Los Centros Docentes. Trabajo Presentado En La Xxii Semana De Educación De La Fundación Santillana. Extraído El 12 De Junio De 2010 Desde Http://Dewey.Uab.Es/Pmarques/Docs/Santillana2007.Pdf

Marien, M. (2006). Sinfonía Memo Marien. Node. Infonomia. Extraído El 12 De Junio De 2010 Desde Http://Www.Infonomia.Com/Renacer/06/Fitxes/Fitxa_Mmarien.Php

Ministerio De Educación Y Ciencia. Ministerio De Industria, Turismo Y Comercio.

Red.Es. Plan Avanza. (2006). «Las Tecnologías De La Información Y La Comunicación A La Educación. Informe Sobre Implantación Y Uso De Las Tic En Educación Primaria I Secundaria (Curso 2005--06)». Extraído El 12 De Junio De 2010 Desde Http://Www.Oei.Es/Tic/Ticcd.Pdf

Pérez, M. A.; Aguaded, J. I. Y Fandos, M. (2009) «Una Política Acertada Y La Formación Permanente Del Profesorado, Claves En El Impulso De Los Centros Tic De Andalucía (España)». Edutec, Revista Electrónica De Tecnología Educativa. Núm. 29/ Julio 2009. Extraído El 25 De Junio De 2010 Desde Http://Edutec.Rediris.Es/Revelec2/Revelec29/Edutec29_Formacion_Permamente_Profesorad O_Tic_Andalucia.Html

Salomé, T. (2010). «Uso De Las Tic En La Práctica Docente De Los Maestros De Educación Básica Y Bachillerato De La Ciudad De La Loja ». Edutec: Revista Electrónica De Tecnología Educativa (32), 1--16. Extraído El 15 De Noviembre De 2010 Desde Http://Edutec.Rediris.Es/Revelec2/Revelec33/ Issn1135---9250.

Sigalés, C., Monimó, J.M. Y Otros (2008). La Integración De Internet En La Educación Escolar Española: Situación Actual Y Perspectivas De Futuro. Ariel: Editorial Uoc; Extraído El 25 De Junio De 2010 Desde Http://Www.Uoc.Edu/In3/Integracion_Internet_Educacion_Escolar/Esp/Pdf/Capitulo10.Pdf

Vivancos, J. (2006). L’Cordinador Tansparent. Bloc Tic---Tac. Extraído 25 De Junio De 2010 Desde Http://Ticotac.Blogspot.Com/2006_12_01_Archive.Html

Vivancos, J. (2008). Tratamiento De La Información Y Competencia Digital. Madrid: Alianza Editorial. 